\title{
Changes in cytochrome P450s-mediated drug clearance in patients with hepatocellular carcinoma in vitro and in vivo: a bottom-up approach
}

\author{
Jie Gao ${ }^{1, *}$, Jun Zhou ${ }^{1, *}$, Xiao-Pei He ${ }^{1}$, Yun-Fei Zhang ${ }^{1}$, Na Gao ${ }^{1}$, Xin Tian ${ }^{1}$, Yan Fang ${ }^{1}$, \\ Qiang Wen ${ }^{1}$, Lin-Jing Jia ${ }^{1}$, Han Jin ${ }^{1}$, Hai-Ling Qiao ${ }^{1}$ \\ ${ }^{1}$ Institute of Clinical Pharmacology, Zhengzhou University, Zhengzhou, China \\ *These authors have contributed equally to this work \\ Correspondence to: Hai-Ling Qiao, e-mail: qiaoh/@zzu.edu.cn \\ Keywords: hepatocellular carcinoma, bottom up IVIVE approach, hepatic clearance, cytochrome P450s, microsomal protein per \\ gram of liver \\ Received: February 14, 2016 \\ Accepted: March 27, 2016 \\ Published: April 12, 2016
}

\section{ABSTRACT}

Hepatocellular carcinoma (HCC) accompanied by severe liver dysfunction is a serious disease, which results in altered hepatic clearance. Generally, maintenance doses depend upon drug clearance, so individual dosage regimens should be customized for HCC patients based on the condition of patients. Based on clearance of CYP isoform-specific substrates at the microsomal level $\left(\mathrm{CL}_{M}\right)$, microsomal protein per gram of liver (MPPGL), liver weight, hepatic blood flow, hepatic clearance values $\left(\mathrm{CL}_{H}\right)$ for $10 \mathrm{CYPs}$ in HCC patients $(n=102)$ were extrapolated using a predictive bottomup pharmacokinetic model. Compared with controls, the $\mathrm{CL}_{M}$ values for CYP2C9, 2D6, 2E1 were significantly increased in HCC patients. Additionally, CYP1A2, 2C8, 2 C19 $C_{M}$ values decreased while the values for CYP2A6, 2B6, 3A4/5 were unchanged. The MPPGL values in HCC tissues were significantly reduced. $\mathrm{CL}_{H}$ values of $\mathrm{HCC}$ patients for CYP1A2, 2A6, 2B6, 2C8, 2C19, and 3A4/5 were significantly reduced, while this for CYP2E1 were markedly increased and those for CYP2C9 and 2D6 did not change. Moreover, disease (fibrosis and cirrhosis) and polymorphisms of the CYP genes have influenced the $\mathrm{CL}_{\mathrm{H}}$ for some CYPs. Prediction of the effects of HCC on drug clearance may be helpful for the design of clinical studies and the clinical management of drugs in HCC patients.

\section{INTRODUCTION}

Significant research efforts towards the development of personalized medicine have been conducted especially for patients with liver diseases that usually are accompanied by the loss of functional hepatocytes. Because of the complex pharmacokinetics changes that occur in liver diseases, both the US FDA and European Medicines Agency have released general guidelines that recommend conducting pharmacokinetic studies when drugs are likely to be used in patients with impaired hepatic function.

Hepatocellular carcinoma (HCC) is the most common type of liver cancer and represents a leading cause of cancer death worldwide [1]. HCC often results in biochemical and physiological changes, such as altered hepatic function, hepatic blood flow $\left(\mathrm{Q}_{\mathrm{H}}\right)$, functional liver size, and plasma protein binding. These changes can result in changed clearance compared with what is observed in subjects with normal hepatic function. Generally, maintenance doses depend on drug clearance, so individual dosage regimens should be customized for HCC patients based on the condition of each patient.

Cytochrome P450 (CYP) represents a large group of enzymes that localize to the endoplasmic reticulum and play critical roles in the metabolism of endogenous and exogenous molecules, including most carcinogens, procarcinogens and drugs [2]. Previous studies of CYP have found that the clearance for CYP3A4 in tumor tissues from $\mathrm{HCC}$ patients was significantly reduced compared with adjacent non-cancerous tissues [3]. Additionally, in vitro studies have indicated that clearance values for CYPs were selectively altered in the presence of cirrhosis $[4,5]$. Moreover, previously published pharmacokinetic 
studies have demonstrated that clearance for CYP3A4/5 was markedly decreased in patients with either cirrhosis [6] or severe alcoholic cirrhosis [7], while the clearance for CYP2C19 was also significantly reduced in patients with liver cirrhosis [8]. Therefore, assessments of changes in clearance values for CYPs may be useful not only for designing personalized HCC treatments, but also for identifying dosage regimens for drugs that are used to treat HCC patients who suffer from other diseases. However, studies that base dosage adjustments on changes in clearance values for CYPs in HCC patients have not been previously reported.

To customize individual dosage regimens for HCC patients with scarce or imprecise available data, in vitro studies using human liver microsomes (HLMs) from the patients combined with predictive bottom-up pharmacokinetic models should be employed. A study by Johnson et al. suggested that $\mathrm{Q}_{\mathrm{H}}$, functional liver size, and plasma protein binding were altered in correlation with the severity of liver cirrhosis [9]. Therefore, changes in these parameters for HCC patients might result in different changes in drugs clearance, both in vitro and in vivo, compared with controls. Indeed, other than $\mathrm{Q}_{\mathrm{H}}$, functional liver size, and plasma protein binding, the change in microsomal protein per gram of liver (MPPGL) has been found to be a more important parameter. Unfortunately, data regarding MPPGL values in patients with liver cirrhosis or HCC have not been reported, which represents a serious obstacle to determining the $\mathrm{CL}_{\mathrm{H}}$ in $\mathrm{HCC}$ patients.

Accordingly, an in vitro study of $102 \mathrm{HCC}$ patient samples was performed that focused on the clearance changes for 10 CYPs-CYP1A2, 2A6, 2B6, 2C8, 2C9, 2C19, 2D6, 2E1, and 3A4/5. Relevant physiological and biochemical changes related to liver disease were incorporated into predictive bottom-up pharmacokinetic models to analyze changes in clearance at different levels. The effects of an accompanying disease (i.e., cirrhosis and/or fibrosis), genetic polymorphisms, and demography on hepatic clearance were evaluated. We hope the findings of this study could be applied to guide appropriate trial design for population pharmacokinetic studies or the clinical management of drugs in HCC patients in whom no clinical data exist.

\section{RESULTS}

\section{Clearance at the microsomal level}

Clearance determined based on "per $\mathrm{mg}$ of microsomal protein" was considered as $\mathrm{CL}_{\mathrm{M}}$, which was calculated based on the ratio of $\mathrm{V}_{\max }$ to $\mathrm{K}_{\mathrm{m}}$. The $\mathrm{CL}_{\mathrm{M}}$ values for 10 CYPs (CYP1A2, 2A6, 2B6, 2C8, 2C9, 2C19, 2D6, $2 \mathrm{E} 1$, and $3 \mathrm{~A} 4 / 5)$ were measured in HLMs from both HCC groups and controls; the results are shown in Figure 1.

Compared with controls, the $\mathrm{CL}_{\mathrm{M}}$ values for CYP2C9, 2D6, and 2E1 increased in HCC patients
( $P=7.96 \mathrm{E}-5,2.91 \mathrm{E}-3$, and $1.56 \mathrm{E}-21$, respectively), while those values for CYP1A2, 2C8, and 2C19 were lower ( $P=9.61 \mathrm{E}-9,1.19 \mathrm{E}-24$, and 3.61E-4, respectively). The $\mathrm{CL}_{\mathrm{M}}$ values for $2 \mathrm{~A} 6,2 \mathrm{~B} 6$, and CYP3A4/5 were unchanged. Notably, the most marked increase was the $\mathrm{CL}_{\mathrm{M}}$ value for CYP2E1, which increased by $99.5 \%$, while the most prominent reduction was the $\mathrm{CL}_{\mathrm{M}}$ for $\mathrm{CYP} 2 \mathrm{C} 8$, which declined by $77 \%$.

There was great intra-individual variation in the $\mathrm{CL}_{\mathrm{M}}$ values for CYPs. Notably, the ratios of maximum to minimum were $147.7,1000.0,89.0,100.0,56.3,17.7$, 111.6, 13.1, and 14.9 for the $\mathrm{CL}_{\mathrm{M}}$ values for CYP1A2, 2A6, 2B6, 2C8, 2C9, 2C19, 2D6, 2E1, and 3A4/5.

\section{Clearance at the liver tissue level}

The MPPGL values [28.85 (7.60-93.60) mg/g] were initially determined in $102 \mathrm{HCC}$ patients. The MPPGL values in $\mathrm{HCC}$ patients were not normally distributed and were significantly lower $(P=1.48 \mathrm{E}-5)$ than the values in control patients[39.60 (9.90-127.90) $\mathrm{mg} / \mathrm{g}]$ [10].

According to the contents of MPPGL, individual clearance of liver tissue $\left(\mathrm{CL}_{\mathrm{LT}}\right)$ for CYPs was calculated by multiplying each individual MPPGL by the corresponding individual $\mathrm{CL}_{\mathrm{M}}$ for CYPs, which represents CYP-mediated clearance in liver tissue. As shown in Figure 1, the $\mathrm{CL}_{\mathrm{LT}}$ values for CYP1A2, 2A6, 2B6, 2C8, 2C19, and 3A4/5 in HCC patients were dramatically lower than in controls $(P=2.00 \mathrm{E}-11,7.88 \mathrm{E}-5,1.37 \mathrm{E}-2,8.86 \mathrm{E}-26,1.01 \mathrm{E}-7$, and $1.37 \mathrm{E}-2$, respectively). Notably, the $\mathrm{CL}_{\mathrm{LT}}$ for $\mathrm{CYP} 2 \mathrm{E} 1$ was significantly greater $(P=3.12 \mathrm{E}-6)$. However, the $\mathrm{CL}_{\mathrm{LT}}$ for CYP2C9 and 2D6 showed no significant difference. Overall, the $\mathrm{CL}_{\mathrm{LT}}$ for most CYPs were significantly lower in HCC patients.

\section{Clearance at the liver level}

The mean body weight (BW) of the 105 control cases was $63.96(30.00-92.00) \mathrm{kg}$, and the mean liver weight (LW) calculated based on the BW of these cases was 1337.24 (912.31-1688.09) g. The mean BW and LW of the102 HCC patients were $66.00(40.00-101.00) \mathrm{kg}$ and 1103.84 (840.32-1458.57) g, respectively. The LW of the HCC patients was significantly lower than that of the controls ( $P=1.34 \mathrm{E}-11)$, while the $\mathrm{BW}$ of two groups showed no significant difference.

According to the $\mathrm{BW}$ and $\mathrm{LW}$ values determined above, individual clearance in the liver $\left(\mathrm{CL}_{\mathrm{L}}\right)$ values were obtained by multiplying each individual LW/BW by the individual $\mathrm{CL}_{\mathrm{LT}}$, which represents CYP-mediated clearance in the liver. As shown in Figure 1, the $\mathrm{CL}_{L}$ values for CYP1A2, 2A6, 2B6, 2C8, 2C19, and 3A4/5 in $\mathrm{HCC}$ patients were dramatically lower than in controls $(P=1.04 \mathrm{E}-13,1.92 \mathrm{E}-7,8.99 \mathrm{E}-4,3.77 \mathrm{E}-27,6.71 \mathrm{E}-10$, and $3.10 \mathrm{E}-4$, respectively), while the $\mathrm{CL}_{\mathrm{L}}$ value for CYP2E1 was higher $(P=1.27 \mathrm{E}-3)$ and the $\mathrm{CL}_{\mathrm{L}}$ value for CYP2C9 
A
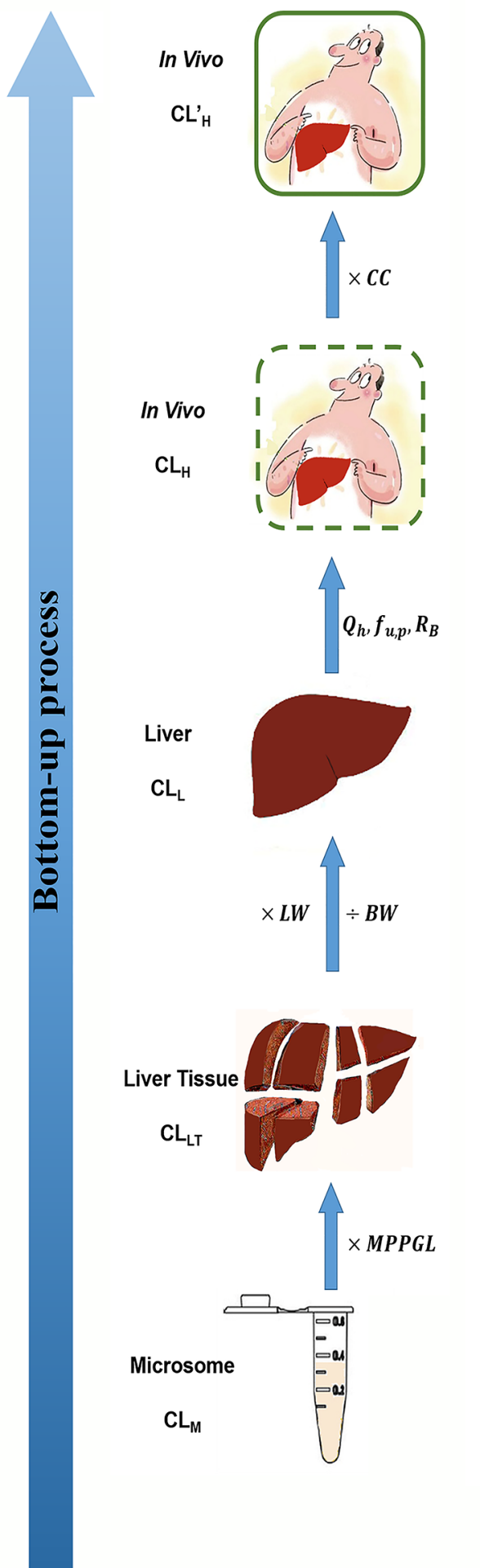
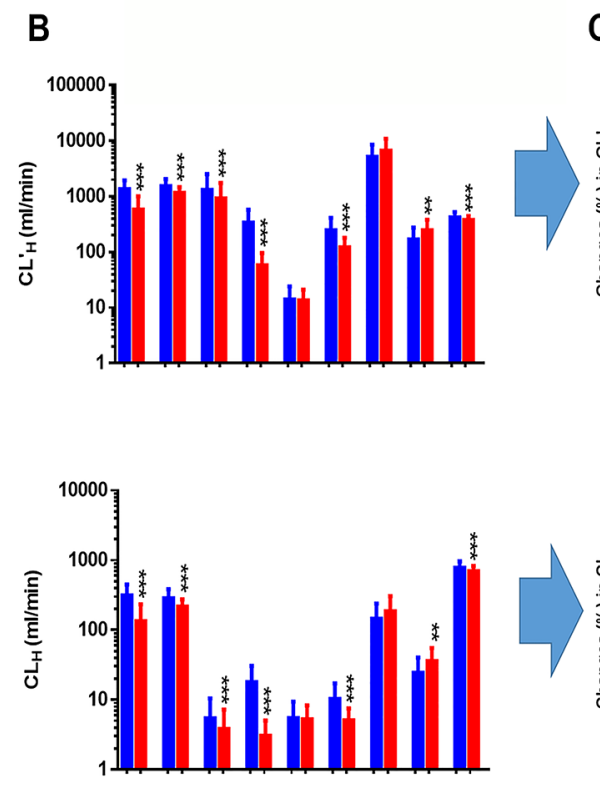

C
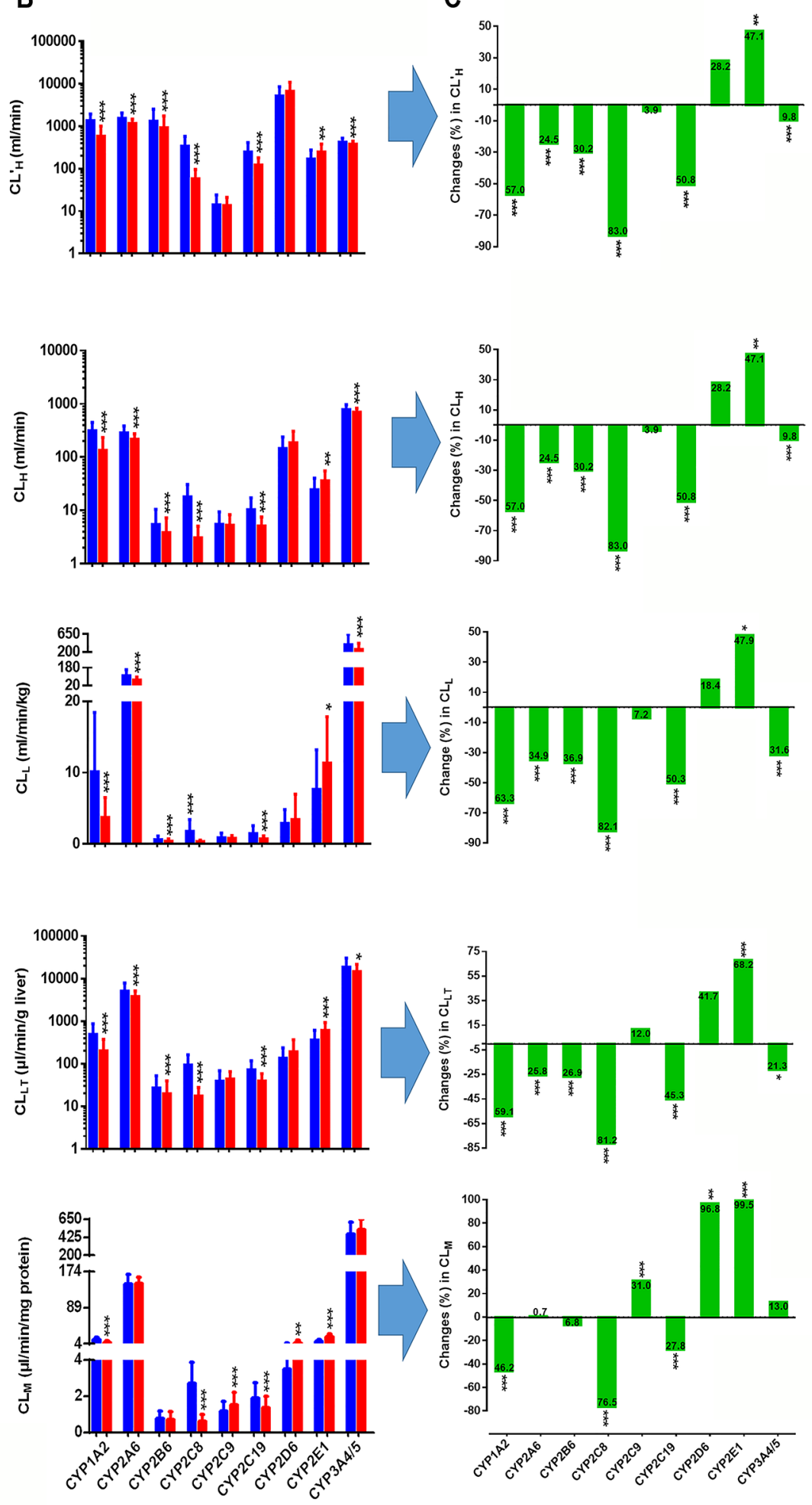

Figure 1: The bottom-up process (A), Clearance for CYPs at different levels in hepatocellular carcinoma (HCC) patients and control subjects $(B)$ and changes in clearance rates at different levels in $\mathrm{HCC}$ patients $(C) . \mathrm{CL}_{\mathrm{M}}$ : clearance at the microsomal level; MPPGL: microsomal protein per gram of liver; $\mathrm{CL}_{\mathrm{LT}}$ : clearance at the liver tissue level; $\mathrm{LW}$ : liver weight; $\mathrm{BW}$ : body weight; $\mathrm{CL}_{\mathrm{L}}$ : clearance at the liver level; $\mathrm{Q}_{\mathrm{H}}$ : hepatic blood flow; $\mathrm{f}_{\mathrm{u}, \mathrm{p}}$ : fraction unbound in plasma; $\mathrm{R}_{\mathrm{B}}$, ratio of the drug concentration in blood to plasma; $\mathrm{CL}_{\mathrm{H}}$ : clearance in vivo; $\mathrm{CC}$ : correction coefficient; $\mathrm{CL}_{\mathrm{H}}$ : corrected clearance in vivo. The clearance for $\mathrm{CYPs}$ are expressed as medians with the inter-quartile range. A blue bar represents clearance in controls; a red bar represents clearance in the HCC group. "**", “**”, and “***” indicate significant differences from controls $(P<0.05, P<0.01$, and $P<0.001$, respectively) by the Mann-Whitney U test. 
and 2D6 showed no significant difference. At the liver level, most $C Y P \mathrm{CL}_{\mathrm{L}}$ values were also markedly lower in HCC patients.

\section{In vivo clearance}

The mean $\mathrm{C}_{\mathrm{O}}$ value for control cases, which was determined based on age and gender, was 5.14 (4.92-6.65) $\mathrm{L} / \mathrm{min}$. The mean $\mathrm{Q}_{\mathrm{H}}$ value calculated based on the $\mathrm{C}_{\mathrm{O}}$ was $1259.30(1205.40-1629.25) \mathrm{ml} / \mathrm{min}$. The mean $\mathrm{C}_{\mathrm{O}}$ and $\mathrm{Q}_{\mathrm{H}}$ values in HCC patients of $5.83(4.92-6.65) \mathrm{L} / \mathrm{min}$ and $1428.35(1205.40-1629.25) \mathrm{ml} / \mathrm{min}$ were significantly higher than those in controls $(P=1.26 \mathrm{E}-10$ and $1.29 \mathrm{E}-10$, respectively).

Using the conventional in vitro-in vivo extrapolation (IVIVE) method, clearance at the in vivo level or hepatic clearance $\left(\mathrm{CL}_{\mathrm{H}}\right)$ for CYPs in controlled cases and HCC patients were predicted; results are shown in Figure 1. Compared with controls, only the $\mathrm{CL}_{\mathrm{H}}$ for CYP2E1 in HCC patients was significantly increased $(P=1.28 \mathrm{E}-4)$, while the $\mathrm{CL}_{\mathrm{H}}$ values for CYP1A2, 2A6, 2B6, 2C8, 2C19, and $3 \mathrm{~A} 4 / 5$ were significantly reduced $(P=6.14 \mathrm{E}-13,3.86 \mathrm{E}-$ $6,1.46 \mathrm{E}-3,9.98 \mathrm{E}-27,1.87 \mathrm{E}-9$, and $1.30 \mathrm{E}-2$, respectively) and the $\mathrm{CL}_{\mathrm{H}}$ for CYP2C9 and 2D6 did not significantly change.

To evaluate the predictive performance, the accuracy of the predicted $\mathrm{CL}_{\mathrm{H}}$ values for CYPs in control cases were compared with the observed clearance in vivo; results are shown in Figure 2. The AFEs were 0.231 for CYP1A2, 0.186 for CYP2A6, 0.007 for CYP2B6, 0.053 for CYP2C8, 0.391 for CYP2C9, 0.042 for CYP2C19, 0.028 for CYP2D6, 0.241 for CYP2E1, and 1.854 for CYP3A4/5, which demonstrated that only the $\mathrm{CL}_{\mathrm{H}}$ for CYP3A4/5 was correct. To test the accuracy of each individual measurement, the IFE was calculated. Our findings suggested that the $\mathrm{CL}_{\mathrm{H}}$ value for CYP3A4/5 matched most closely with its clearance in vivo, for which $58(55.2 \%)$ of the cases were within a 2-fold error range. After CYP3A4/5, the $\mathrm{CL}_{\mathrm{H}}$ value for CYP2C9 matched best with its clearance in vivo, for which 28 (26.7\%) of the cases were within a 2-fold error range.

No data on the in vivo clearance for CYPs in HCC patients was identified in a literature search, and only data regarding the clearance for CYP2C19 $(67 \pm 40 \mathrm{ml} / \mathrm{min})$ and CYP3A4/5 (319.49 $\pm 154.42 \mathrm{ml} / \mathrm{min})$ in liver cirrhosis were queried. Therefore, the $\mathrm{CL}_{\mathrm{H}}$ values for CYP2C19 and $3 \mathrm{~A} 4 / 5$ predicted using the conventional IVIVE method for HCC patients were compared with the observed clearance in cirrhotic patients; our findings revealed that the AFE values for CYP2C19 and 3A4/5 were 0.087 and 2.111, respectively.

To obtain more accurate measurements of the $\mathrm{CL}_{\mathrm{H}}$ value for CYPs, the CCs of different CYPs were introduced into the conventional IVIVE method. The CC was the inverse of the corresponding AFE. For control cases, the CC was 4.334 for CYP1A2, 5.369 for CYP2A6, 139.371 for CYP2B6, 18.938 for CYP2C8, 2.558 for
CYP2C9, 24.026 for CYP2C19, 35.791 for CYP2D6, 4.152 for CYP2E1, and 0.540 for CYP3A4/5. For HCC patients, the CC was 11.524 for CYP2C19 and 0.474 for CYP3A4/5. Figure 2 shows that the corrected predictive hepatic clearance $\left(\mathrm{CL}_{\mathrm{H}}\right)$ value for all CYPs for control cases had better accuracy than the $\mathrm{CL}_{\mathrm{H}}$ values compared with observed clearance rates in vivo.

Based solely on clearance data for CYP2C19 and $3 \mathrm{~A} 4 / 5$ in cirrhotic patients identified in a literature search, CCs that were suitable for controls and one that was calculated based on clearance in cirrhotic patients were introduced into the conventional method to analyze whether the CCs applied to controls were also suitable for HCC patients. Cross tabs with $\chi^{2}$ tests for independence analyses revealed that there were no significant differences between the HCC and control groups; results are shown in Table 1. From the examples of these two CYPs, one conclusion that might be tentatively drawn is that the CCs that were applied to control cases also were suitable for HCC patients.

The $\mathrm{CL}_{\mathrm{H}}$ values for CYPs in $\mathrm{HCC}$ patients are shown in Figure 1. The $\mathrm{CL}_{\mathrm{H}}$, values for CYP1A2, 2A6, $2 \mathrm{~B} 6,2 \mathrm{C} 8,2 \mathrm{C} 19$, and $3 \mathrm{~A} 4 / 5$ were significantly reduced $(P=6.14 \mathrm{E}-13,3.86 \mathrm{E}-6,1.46 \mathrm{E}-3,9.98 \mathrm{E}-27,1.87 \mathrm{E}-9$, and $1.30 \mathrm{E}-2$, respectively). The most prominent reduction was observed for the $\mathrm{CL}_{\mathrm{H}}$, value for $\mathrm{CYP} 2 \mathrm{C} 8$, which was reduced by $84.8 \%$. Following CYP2C 8 were CYP1A2, $2 \mathrm{C} 19,2 \mathrm{~B} 6,2 \mathrm{~A} 6,3 \mathrm{~A} 4 / 5$, and $2 \mathrm{C} 9$, for which the $\mathrm{CL}_{\mathrm{H}}$ values were reduced by $60.7 \%, 57.2 \%, 43.9 \%, 38.0 \%$, $19.6 \%$, and $15.7 \%$, respectively. Compared with controls, the $\mathrm{CL}_{\mathrm{H}}$, values for CYP2E1 and 2D6 increased by $40.3 \%$ and $11.9 \%$, respectively, although it should be noted that the $\mathrm{CL}_{\mathrm{H}}$, values for $\mathrm{CYP} 2 \mathrm{C}$ 9 and 2D6 showed no significant differences.

For $\mathrm{CL}_{\mathrm{H}}{ }_{\mathrm{H}}$ values, obvious intra-individual variation could be observed. The maximum to minimum ratios were 83.9, 1036.7, 183.2, 147.4, 56.5, 73.8, 175.7, 60.6, and 11.5 for CYP1A2, 2A6, 2B6, 2C8, 2C9, 2C19, 2D6, 2E1 and $3 \mathrm{~A} 4 / 5$, respectively; among these CYPs, CYP2A6 exhibited the greatest intra-individual variation.

\section{Bottom-up changes in CYP clearance}

Figure 1 illustrates bottom-up changes in CYP clearance both in vitro and in vivo in patients with HCC compared with control patients. At each step, changes in the clearance values for CYPs differed. Notably, at each level (microsome, liver tissue, liver, and in vivo), CYP2E1 clearance was significantly increased, while the clearance values for CYP1A2, 2C8, and 2C19 were significantly reduced. The clearance values for CYP2A6, 2B6, and $3 \mathrm{~A} 4 / 5$ showed no change at the microsomal level, although the clearance values at the other three levels were significantly reduced. The clearance values for CYP2C9 and 2D6 at the microsomal level were markedly increased, while those at the other three levels showed no change. 


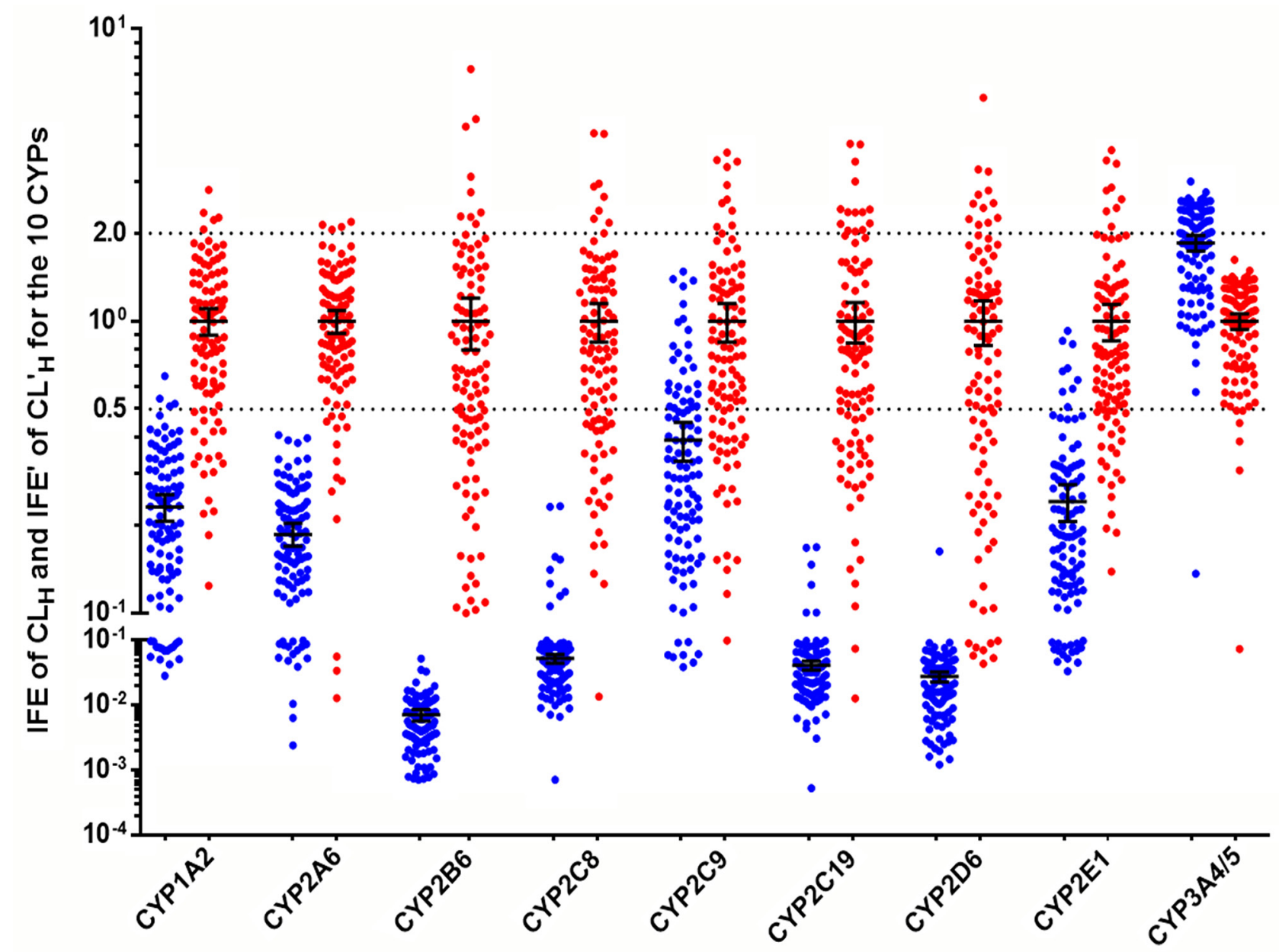

Figure 2: Individual fold-error(IFE) of CLH and corrected individual fold-error(IFE') of CL'H for the 10 CYPs in control subjects $(\mathbf{n}=\mathbf{1 0 5}) . \quad$ IFE $=10^{\left.\frac{1}{N} \sum \log \text { (predicted individual value/observed overall mean }\right)} ; \mathrm{IFE}^{\prime}=10^{\frac{1}{N} \sum \log (\text { corrected predictive individual value/observed overall mean })}$. The blue ball represents the IFE, the red ball represents the IFE'. The black horizontal lines represent the mean with $95 \%$ confidence interval.

Table 1: Comparison of the accuracy of predictions using different in vivo data

\begin{tabular}{lcccccccc}
\hline Drug & Group & \multicolumn{2}{c}{ Within a 2-fold error } & & \multicolumn{2}{c}{ Outside a 2-fold error } & \multirow{2}{*}{$\chi^{2} \boldsymbol{P}$-value } \\
\cline { 3 - 4 } & & Number & Percentage & & Number & Percentage & \\
\hline \multirow{2}{*}{ Omeprazole } & Control & 55 & 53.92 & & 47 & 46.08 & 0.480 \\
& HCC & 60 & 58.82 & & 42 & 41.18 & \\
\multirow{2}{*}{ Midazolam } & Control & 95 & 93.14 & & 7 & 6.86 & 1.000 \\
& HCC & 95 & 93.14 & & 7 & 6.86 & \\
\hline
\end{tabular}

Control: using a correction coefficient that applies to patients with normal liver function. HCC: using a correction coefficient calculated based on in vivo clearance in patients with cirrhosis. 


\section{Factors that impact the in vivo clearance}

To further assess the effects of disease progression on in vivo clearance, HCC patients were categorized into fibrosis and cirrhosis subgroups based on their histological diagnosis. The results found that only the $\mathrm{CL}_{\mathrm{H}}$ for CYP2D6 was significantly different between the fibrosis and cirrhosis groups, and was increased by $88.3 \%$ in patients with cirrhosis (Figure 3A).

The impact of genetic polymorphisms on the in vivo clearance for CYPs was also assessed; no genetic influence and less than 7 polymorphic individuals were not displayed. As shown in (Figure 3B-3F), the $\mathrm{CL}_{\mathrm{H}}$ value for CYP1A2 was influenced by CYP1A2 $(-163 C>A)$. The $\mathrm{CL}_{\mathrm{H}}$ values for CYP2B6 differed by the genotype of CYP2B6 $(516 G>T)$. The $\mathrm{CL}_{\mathrm{H}}$, values for CYP2D6, 2E1, and 3A4/5 were influenced by CYP2D6 (100C $>T), C Y P 2 E 1(-333 T>A)$, and CYP3A5 (6986A>G), respectively.

The influence of gender, age, smoking and alcohol on the in vivo clearance for CYPs was also analyzed and found that smoking and alcohol consumption influenced the $\mathrm{CL}_{\mathrm{H}}$, for CYP2D6, as shown in (Figure $3 \mathrm{G}$ and $3 \mathrm{H}$ ). Compared with nonsmokers, the $\mathrm{CL}_{\mathrm{H}}$ for CYP2D6 was significantly lower for smokers. Alcohol consumption significantly reduced the $\mathrm{CL}_{\mathrm{H}}$ for CYP2D6. Gender and age had no influence on the in vivo clearances for CYPs.

Together, our findings indicated that only $\mathrm{CL}_{\mathrm{H}}$ for CYP2D6 was easily influenced by disease progression, smoking and alcohol consumption, and only genetic mutations in CYP1A2 (-163C $>A), C Y P 2 B 6(516 G>T)$, CYP2D6 (100C $>T), C Y P 2 E 1$ ( $-333 T>A)$, and CYP $3 A 5$ $(6986 A>G)$ had clear effects on the in vivo clearance for corresponding CYP.

\section{DISCUSSION}

This represents the first extensive study to test the clearances for 10 CYPs in vitro and in vivo using 102 liver samples from patients with $\mathrm{HCC}$ accompanied by fibrosis or cirrhosis. We found that compared with controls the $\mathrm{CL}_{\mathrm{M}}$ values increased for CYP2C9, $2 \mathrm{D} 6$ and 2E1, while the values for CYP1A2, 2C8 and 2C19 decreased and the values for CYP2A6, 2B6 and 3A4/5 were similar. MPPGL values corresponding with an unprecedented large number samples were first determined, which were significantly lower compared with controls [28.85 (7.60-93.60) $\mathrm{mg} / \mathrm{g}$ vs $39.60(9.90-127.90) \mathrm{mg} / \mathrm{g}]$ [10]. Based on changes in both physiological (e.g. MPPGL, LW, $\mathrm{C}_{\mathrm{O}}$, and $\mathrm{Q}_{\mathrm{H}}$ ) and enzyme kinetics (e.g. $\mathrm{V}_{\max }, \mathrm{K}_{\mathrm{m}}$ and $\mathrm{CL}_{\mathrm{M}}$ ) parameters, the altered in vivo clearances for CYPs were identified. Compared with controls, the in vivo clearances for CYP1A2, 2A6, $2 \mathrm{~B} 6,2 \mathrm{C} 8,2 \mathrm{C} 19,3 \mathrm{~A} 4 / 5$ were significantly decreased, the clearance for CYP2E1 was markedly increased, while the clearances for CYP2C9 and 2D6 showed no change.

Using previously published data from other groups, an attempt to predict the pharmacokinetics of three drugs in liver cirrhosis had been performed [11]. Similarly, another previously attempt collected a series of parameters (e.g. $\mathrm{CL}_{\mathrm{M}}, \mathrm{V}_{\max }, \mathrm{K}_{\mathrm{m}}$, and MPPGL) reported in the literature to predict the effects of liver cirrhosis on drug clearance [9]. Herein, we report the first attempt to predict the clearances for CYPs at different levels (e.g., liver tissue, liver, and in vivo) in HCC patients and to investigate the effects of HCC on the clearances for CYPs at different levels. Additionally, in our present study, we performed in vitro studies of each individual using 102 HLMs of HCC patients, determined the MPPGL of each individual, and recorded and saved other physiological and pathological parameters from each patient. Therefore, we could not only explore the reasons for these changes, but also revealed intra-individual variations in the clearances for CYPs at the microsome, liver tissue, liver, and in vivo levels.

Several previous studies have reported that the clearance values for several CYPs-CYP1A2, 2A6, 2D6, $2 \mathrm{C} 19,2 \mathrm{E} 1$ and $3 \mathrm{~A}-$ were decreased $[5,12-17]$. However, we found that the $\mathrm{CL}_{\mathrm{M}}$ values for CYP2C9, 2D6 and 2E1 were significantly greater. This inconsistency could be due in part to the fact that these earlier studies focused on patients with simple cirrhosis who did not have accompanying HCC. Additionally, another study found that CYP2A6 clearance was reduced in patients with either moderate or severe alcoholic liver disease, but not in those with only mild disease [17]. By contrast, our present study showed that CYP2A6 clearances were not altered in the fibrosis or cirrhosis subgroups of HCC patients. These different characteristics identified in our present study suggest that a special reference standard may be essential for personalizing treatments of $\mathrm{HCC}$ patients with cirrhosis.

To the best of our knowledge, this present study was the first extensive to measure microsomal protein content in a large number of HCC patients. Compared with controls [39.60 (9.90-127.90) mg/g] [10], the MPPGL values $[28.85(7.60-93.60) \mathrm{mg} / \mathrm{g}]$ were significantly reduced in HCC patients $(P=1.48 \mathrm{E}-5)$. Because of the reduced MPPGL, the $\mathrm{CL}_{\mathrm{M}}$ for $\mathrm{CYP} 2 \mathrm{~A} 6,2 \mathrm{~B} 6$, and $3 \mathrm{~A} 4 / 5$ showed no change, but the $\mathrm{CL}_{\mathrm{LT}}$ values were significantly reduced. The $\mathrm{CL}_{\mathrm{M}}$ values for $\mathrm{CYP} 2 \mathrm{C} 9$ and $2 \mathrm{D} 6$ were markedly increased, while that of $\mathrm{CL}_{\mathrm{LT}}$ showed no change. Meanwhile, because of the markedly reduced LW in HCC patients, the $\mathrm{CL}_{\mathrm{L}}$ was more significantly reduced compared with the $\mathrm{CL}_{\mathrm{LT}}$. However, because of the elevated $\mathrm{C}_{\mathrm{O}}$ and $\mathrm{Q}_{\mathrm{H}}$ of $\mathrm{HCC}$ patients, the $\mathrm{CL}_{\mathrm{H}}$ was less significantly reduced compared with the $\mathrm{CL}_{\mathrm{L}}$. As a result of this bottom-up approach, a greater understanding of the changes in clearance values for CYPs at various levels in HCC patients became possible.

The effects of CYP genetic polymorphisms on the clearances for CYPs in vivo were analyzed and we found that only 5 of 24 mutations, including CYP1A2 $(-163 C>A), C T P 2 B 6(516 G>T), C Y P 2 D 6 \quad(100 C>T)$, 
CYP2E1 (-333T>A) and CYP3A5 $(6986 A>G)$ had clear effects on the clearances for CYPs in vivo.

To further verify the factors that influence CYPs clearances in vivo in HCC, we investigated the effects of general factors on CYP clearances. We found no obvious effect of gender and age on all CYPs clearances in vivo, no obvious effect of smoking and drinking on most CYP clearances, with the exceptions of CYP2D6. Although the $\mathrm{CL}_{\mathrm{H}}$ value for CYP2D6 were higher in non-smokers and non-drinkers, these factors were not likely to be related to clearance changes in patients. Indeed, the HCC group in this present study included more smokers and drinkers compared with the control group, and the $\mathrm{CL}_{\mathrm{H}}$, value for CYP2D6 for these patients were higher.

In summary, the bottom-up approach allowed us to predict pharmacokinetics in patients with $\mathrm{HCC}$ accompanied by fibrosis or cirrhosis and explored the reasons for changes in CYPs clearance at different levels. Because dosing recommendations are extremely limited and imprecise in HCC patients, this present
A
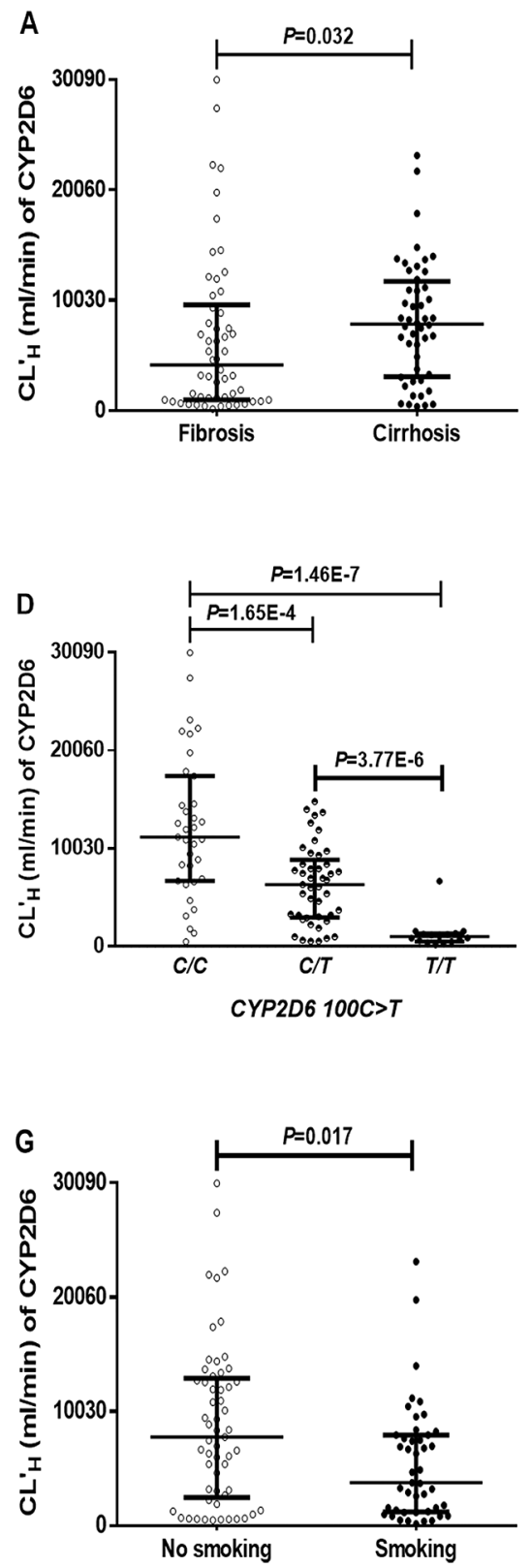
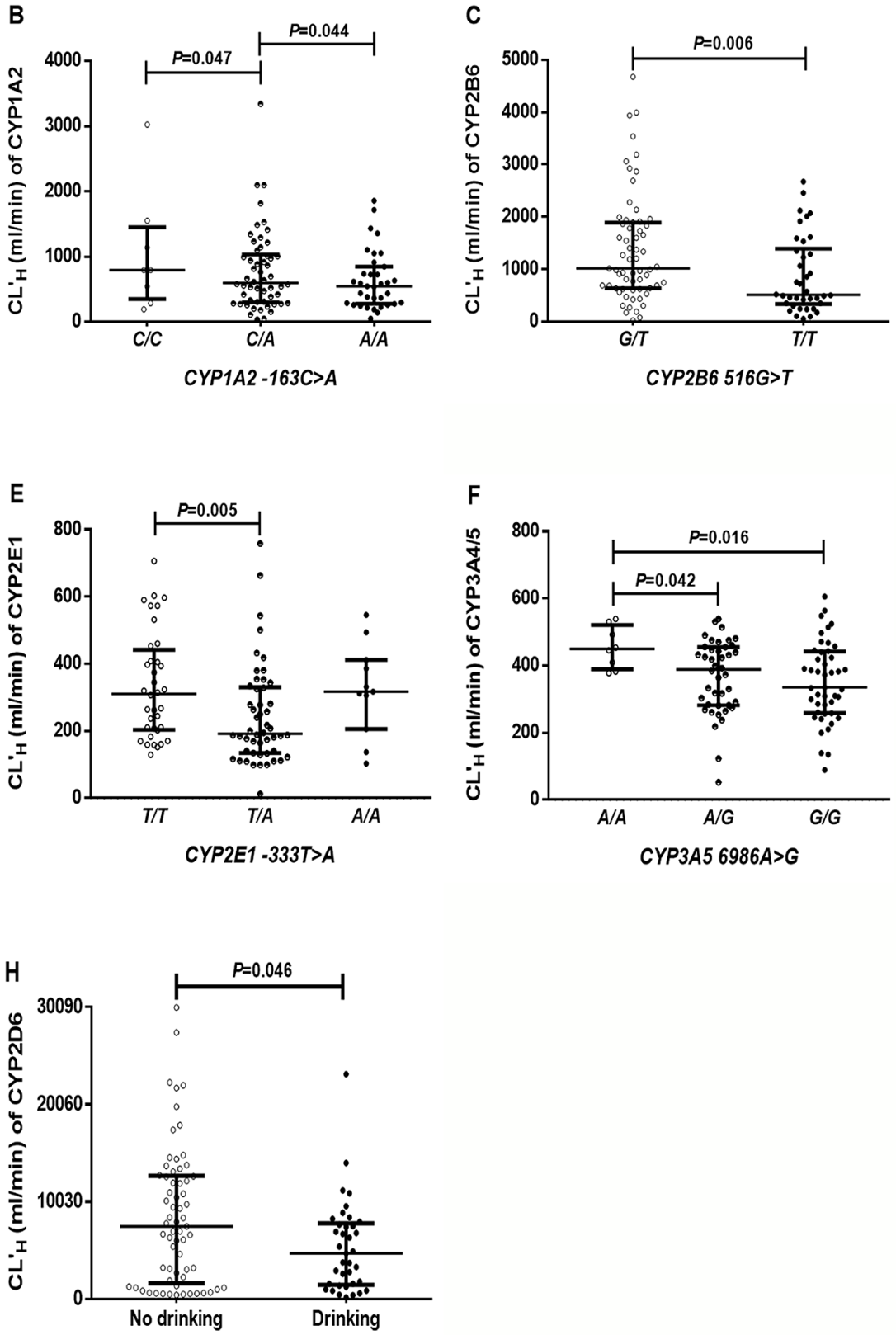

Figure 3: The effects of disease (A), genetic polymorphisms (B-F), smoking (G), and drinking (H), on the corrected predictive hepatic clearance $\left(\mathbf{C L}_{\mathbf{H}}\right)$ ) for $\mathbf{C Y P s}$ in $\mathrm{HCC}$ patients $(\mathbf{n}=\mathbf{1 0 2})$. Black horizontal lines represent medians with the interquartile range. Disease (fibrosis, $\mathrm{n}=54$; cirrhosis, $\mathrm{n}=48) ; C Y P 1 A 2-163 C>A(C / C, \mathrm{n}=8 ; C / A, \mathrm{n}=54 ; A / A, \mathrm{n}=35) ; C Y P 2 B 6516 G>T(G / T$, $\mathrm{n}=63 ; T / T, \mathrm{n}=38) ; C Y P 2 D 6100 C>T(C / C, \mathrm{n}=35 ; C / T, \mathrm{n}=45 ; T / T, \mathrm{n}=16) ; C Y P 2 E 1-333 T>A(T / T, \mathrm{n}=36 ; T / A, \mathrm{n}=50 ; A / A, \mathrm{n}=11) ; C Y P 3 A 5$ $6986 A>G(A / A, \mathrm{n}=8 ; A / G, \mathrm{n}=42 ; G / G, \mathrm{n}=45)$; smoking (no smoking, $\mathrm{n}=57$; smoking, $\mathrm{n}=45)$; alcohol (no drinking, $\mathrm{n}=65 ;$ drinking, $\mathrm{n}=37$ ). 
study may provide important data to help clinicians to adjust their prescriptions for a wide range of drugs that are administrated to $\mathrm{HCC}$ patients. Because of the dramatically increased clearance for CYP2E1 in HCC patients, which metabolizes nitrosamine compounds into strong carcinogens, our observations may also aid further studies of the mechanisms of hepatocarcinogenesis in HCC patients. Because the clearance values in vivo for CYPs in the present paper is predicted, the accuracy of these predictions needs to be validated in future studies in HCC patients.

\section{MATERIALS AND METHODS}

\section{Human liver samples}

All non-tumor liver tissues were obtained from patients who had undergone surgical resection at Henan Provincal People's Hospital or Henan Provincal Tumor Hospital between March 2012 and July 2014; informed consent was obtained from each patient. Approvals for tissue collection and in vitro metabolism studies were obtained from the Medical Ethics Committee of Zhengzhou University. This research was conducted in accordance with the Declaration of Helsinki

$\mathrm{HBV}$ - or HCV-infected liver tissues (102 samples) were obtained from patients with HCC confirmed by postoperative pathological examination. Liver samples were classified as either having fibrosis or cirrhosis based on a histological diagnosis. As mentioned previously [18], 105 larger quantity of liver samples derived from 123 liver samples with normal function identified by histological diagnosis were collected from subjects with liver hemangioma, metastatic carcinoma, cholelithiasis, or gallbladder cancer for use as normal liver control tissues.

\section{Microsome preparation}

Human liver microsomes (HLMs) were prepared by differential centrifugation as previously described [10]. Microsomal protein concentrations were measured using the Bradford method [19]. The MPPGL contents were determined as previously described [10].

\section{Clearance at microsomal level $\left(\mathrm{CL}_{\mathrm{M}}\right)$}

The drugs used for the enzymatic assays are probe substrates. Most CYP isoform-specific substrates and metabolite (7'-hydroxycoumarin, bupropion, hydroxybupropion, tolbutamide, 4'-hydroxytolbutamide, omeprazole, 5'-hydroxyomeprazole, dextromethorphan, O-demethylation dextrorphan, chlorzoxazone, 6-hydroxychlorzoxazone, and 1'-hydroxylation midazolam) were purchased from Sigma-Aldrich (St. Louis, MO, USA). Paclitaxel and 6-hydroxylpaclitaxel were purchased from Cayman Co. (Ann Arbor, MI, USA). Phenacetin, acetaminophen, midazolam and coumarin were purchased from the State Food and Drug Administration (Beijing, China). NADPH was obtained from Roche Co. (Basel, Switzerland). All organic solvents were of HPLC grade purity and were obtained from Siyou Chemical Reagent Co. (Tianjin, China).

Incubation mixtures contained HLMs $(0.3 \mathrm{mg}$ protein/ml for CYP1A2, 2A6, and 2E1; $0.2 \mathrm{mg}$ protein/ $\mathrm{ml}$ for CYP2D6 and 3A4/5; $0.5 \mathrm{mg}$ protein/ml for $2 \mathrm{~B} 6$, 2C8, 2C9, and 2C19), $100 \mathrm{mM}$ phosphate buffer ( $\mathrm{pH} 7.4$ ) with $1 \mathrm{mM}$ NADPH and seven or eight concentrations of substrate $(6.25-800 \mu \mathrm{M}$ for phenacetin, $0.156-20 \mu \mathrm{M}$ for coumarin, $7.8-500 \mu \mathrm{M}$ for bupropion, $2.5-80 \mu \mathrm{M}$ for paclitaxel, $31.25-2000 \mu \mathrm{M}$ for tolbutamide, $3.9-500 \mu \mathrm{M}$ for omeprazole, $0.625-960 \mu \mathrm{M}$ for dextromethorphan, $7.8-1000 \mu \mathrm{M}$ for chlorzoxazone, and $3.9-200 \mu \mathrm{M}$ for midazolam). The mixtures were pre-incubated for 5 min at $37^{\circ} \mathrm{C}$. Optimal incubation times were as follows: $30 \mathrm{~min}$ for phenacetin O-deethylation, coumarin 7-hydroxylation, and chlorzoxazone 6-hydroxylation; $60 \mathrm{~min}$ for bupropion 4-hydroxylation, and tolbutamide 4-hydroxylation; 90 min for omeprazole 5-hydroxylation; $120 \mathrm{~min}$ for paclitaxel 6-hydroxylation; $20 \mathrm{~min}$ for dextromethorphan O-demethylation; and $5 \mathrm{~min}$ for midazolam 1'-hydroxylation. Reactions were terminated by adding $20 \mu \mathrm{l}$ ice-cold acetonitrile or $1 \mathrm{ml}$ ethylacetate or perchloric acid. Metabolites were identified by HPLCUV or HPLC-FLD. The Michaelis-Menten constant $\left(\mathrm{K}_{\mathrm{m}}\right)$ and maximum reaction rate $\left(\mathrm{V}_{\max }\right)$ of each CYP were determined by nonlinear regression analysis using GraphPad Prism 6.0 (GraphPad Inc., La Jolla, CA, USA). $\mathrm{CL}_{\mathrm{M}}$ was calculated based on the ratio of $\mathrm{V}_{\max }-\mathrm{to}_{\mathrm{m}}$.

\section{Genotyping}

Genomic DNA was isolated from human liver tissues using a genomic DNA purification kit (QIAGEN Translational Medicine Co., Suzhou, China). Polymorphisms in 10 CYPs with frequencies of more than $1 \%$ in the Chinese sample set were investigated. A total of 20 allelic mutations determined by mass spectrometry and the alleles CYP3A4 (20230G>A), CYP1A2 ($3860 G>A)$, and $C Y P 2 B 6(516 G>T)$ genotyped by PCR sequencing were performed by the LIUHE HUADA Genomics Technology Co., (Beijing, China). The alleles $C Y P 2 E 1 * 1 C / * 1 D$ were genotyped using two-step PCR.

\section{In vivo data collection}

A PUBMED search or articles published from 1975 to 2015 was performed to collect information on the pharmacokinetics of 9 probe drugs for CYPs in humans; detailed information was summarized in Table 2. For the clearance of bupropion and chlorzoxazone, no "intravenous infusion" data was identified, so apparent oral clearance was used. 
Table 2: Detailed data about 9 probe drugs for CYPs

\begin{tabular}{|c|c|c|c|c|c|}
\hline CYP & Drug & Chemical Class & $f_{u, p}$ & $\mathbf{R}_{B}$ & $C L_{\text {in vivo }}(\mathrm{ml} / \mathrm{min})$ \\
\hline $1 \mathrm{~A} 2$ & Phenacetin & $\mathrm{N}$ & $0.594[24]$ & $1[24]$ & $1453.33 \pm 389.24[25]$ \\
\hline $2 \mathrm{~A} 6$ & Coumarin & - & 0.055 & 1 & $1602.5 \pm 547.9[26]$ \\
\hline 2B6 & Bupropion $^{\mathrm{a}}$ & B & $0.150[27]$ & $1[27]$ & $1112.25 \pm 280.26[28]$ \\
\hline $2 \mathrm{C} 8$ & Paclitaxel & - & 0.098 [29] & $0.69[30]$ & $496.42 \pm 210.48[31]$ \\
\hline $2 \mathrm{C} 9$ & Tolbutamide & A & $0.056[32-36]$ & $0.55[37]$ & $19.36 \pm 10.60[32-34]$ \\
\hline $2 \mathrm{C} 19$ & Omeprazole & $\mathrm{N}$ & $0.065[34]$ & $0.60[38]$ & $307.22 \pm 51.52[39,40]$ \\
\hline $2 \mathrm{C} 19$ & Omeprazole $^{\mathrm{b}}$ & $\mathrm{N}$ & $0.065[34]$ & 0.60 [38] & $67.0 \pm 40.0[8]$ \\
\hline 2D6 & Dextromethorphan & B & $0.500[34,37]$ & $0.55[34,37]$ & $6471.67 \pm 5596.67[41]$ \\
\hline $2 \mathrm{E} 1$ & Chlorzoxazone $^{\mathrm{a}}$ & B & $0.028[34]$ & $0.55[34]$ & $131.42 \pm 40.08[42-46]$ \\
\hline $3 \mathrm{~A} 4 / 5$ & Midazolam & $\mathrm{N}$ & $0.042[34,47]$ & $0.54[34,47]$ & $426.65 \pm 95.37[47-49]$ \\
\hline $3 \mathrm{~A} 4 / 5$ & Midazolam $^{\mathrm{b}}$ & $\mathrm{N}$ & $0.042[34,47]$ & $0.54[34,47]$ & $319.49 \pm 154.42[7,15]$ \\
\hline
\end{tabular}

$\mathrm{N}$, neutral compound; $\mathrm{B}$ : basic compound; $\mathrm{A}$ : acidic compound; $\mathrm{f}_{\mathrm{u}, \mathrm{p}}$ : fraction unbound in plasma; $\mathrm{R}_{\mathrm{B}}$ : ratio of the drug concentration in blood to plasma. ${ }^{a}$ : because intravenous infusion method was identified in the search, apparent oral clearance was used orally administered drugs. ${ }^{\text {b: }}$ data of drugs in cirrhotic patients.

If multiple published studies about the plasma unbound fraction $\left(\mathrm{f}_{\mathrm{u}, \mathrm{p}}\right)$, blood-to-plasma concentration ratio $\left(\mathrm{R}_{\mathrm{B}}\right)$, and in vivo clearance for each drug, the overall mean for these data was used in this present paper. To determine the overall variability of clearance in vivo from multiple studies that reported the standard deviation (SD) for each drug, the overall SD was calculated.

The weighted mean (WX) was calculated using equation 1:

$$
\mathrm{WX}=\frac{\sum_{j=1}^{n} n_{j} \times x_{j}}{\sum_{j=1}^{n} n_{j}}
$$

where $n_{j}$ is the number of observations and $x_{j}$ is the mean value from the $\mathrm{j}^{\text {th }}$ study.

An overall SD was calculated from equations 2 and $3:$

Overall SD $=\sqrt{\frac{\text { Overall sum of squares }}{N}}$

O verall.sum of squares $=$

$$
\sum_{j=1}^{n}\left[\left\{\left(S D_{j}\right)^{2}+\left(x_{j}\right)^{2}\right\} \times n_{j}\right]-N \times(W X)^{2}
$$

where $\mathrm{N}$ is the total number of observations from all studies and $\mathrm{SD}_{\mathrm{j}}$ is the $\mathrm{SD}$ from the $\mathrm{j}^{\text {th }}$ study.

\section{Bottom-up calculations of hepatic clearance}

For patients with HCC and controls, the bottomup process using in vitro clearance to extrapolate in vivo clearance included several equations. In particular, the clearance for CYP in liver tissue $\left(\mathrm{CL}_{\mathrm{LT}}\right)$ was calculated using equation 4 :

$$
C L_{L T}=C L_{M} \times M P P G L
$$

The clearance for CYPs in liver $\left(\mathrm{CL}_{\mathrm{L}}\right)$ was determined using equation 5 :

$$
C L_{L}=C L_{L T} \times L W / B W \quad \text { (Eq. 5) }
$$

where LW is liver weight and BW is body weight. According to the actual body weight given for each patient, the LW was calculated by multiplying the liver volume $(\mathrm{LV})$ by the liver density, where $\mathrm{LV}(\mathrm{ml})=12.5$ $\times \mathrm{BW}(\mathrm{kg})+536.4[20]$ and the liver density was 1.001 $\mathrm{g} / \mathrm{ml}$ [21]. For HCC patients, pathology classifications indicated that 54 patients were without cirrhosis and 48 had early stage cirrhosis, so the LV of HCC patients was corrected by multiplying by the reciprocal of the fraction of the control LV (coefficient $=1.12$ ) [9].

The clearance for CYPs in vivo $\left(\mathrm{CL}_{\mathrm{H}}\right)$ was next calculated using the well-stirred model:

$$
C L_{H}=\frac{Q_{H} \times C L_{L} \times f_{u, p} / R_{B}}{Q_{H}+C L_{L} \times f_{u, p} / R_{B}} \quad \text { (Eq. 6) }
$$

where $\mathrm{Q}_{\mathrm{H}}$ is determined as $24.5 \%$ [22] of the cardiac output $\left(\mathrm{C}_{\mathrm{O}}\right) . \mathrm{C}_{\mathrm{O}}$ values originated from data for normal Han Chinese males $(n=783)$ and females $(n=805)$; mean values from each group were selected according to the age and gender of donors used in this study [23]. A study by Johnson et al. concluded that the $\mathrm{Q}_{\mathrm{H}}$ in patients with Child-Pugh scores A for liver cirrhosis had no marked reduction [9]. Thus, the calculation of $\mathrm{Q}_{\mathrm{H}}$ for $\mathrm{HCC}$ patients in this paper was the same as for controls. 
Because individual $\mathrm{CL}_{\mathrm{M}}, \mathrm{MPPGL}, \mathrm{BW}, \mathrm{LW}$, and $\mathrm{Q}_{\mathrm{H}}$ values (both $\mathrm{HCC}$ and control group) were used to predict clearance values for CYPs in vivo, the overall accuracy of the prediction was assessed based on the average folderror (AFE), while the individual accuracy was assessed based on the individual fold-error (IFE). A two-fold bias limit corresponds to $0.5-2.0$ of AFE and IFE values, which were estimated as follows:

$$
\begin{aligned}
& \mathrm{AFE}=10^{\frac{1}{\mathrm{~N}}} \sum \mathrm{bg}(\text { predicted m mean /observed overall mean) } \\
& \mathbb{F E}=10^{\frac{1}{N}} \sum \text { bog (predicted indiridual value/ /bberved overall mean) }
\end{aligned}
$$

where $\mathrm{N}$ is the number of separate reports in the literature concerning intravenous drug clearance, except for bupropion and chlorzoxazone. Because there was no message about individual data in the literature, the observed overall mean was used as a reference was used to calculate the IFE.

To predict the $\mathrm{CL}_{\mathrm{H}}$ for CYPs more accurately, a correction coefficient (CC) was introduced into the conventional in vitro-in vivo extrapolation (IVIVE) method. The CC of one CYP was the inverse of the AFE of the corresponding CYP.

\section{Statistical analysis}

Most data sets were not normally distributed, so nonparametric methods were generally used for statistical analyses. The Mann-Whitney U test was used for pairwise comparisons. A $P$-value $<0.05$ was considered to be statistically significant (two-tailed). SPSS statistics 17.0 software was used for statistical analyses. All graphs were generated using the Adobe Photoshop CC 2014, PowerPoint 2016 and GraphPad Prism version 6.0 software package.

\section{ACKNOWLEDGMENTS}

The authors thank the patients enrolled in the study.

\section{CONFLICTS OF INTEREST}

The authors who have taken part in this study declared that they do not have anything to disclose regarding funding or conflict of interest with respect to this manuscript.

\section{GRANT SUPPORT}

This work was supported by the National Natural Science Foundation of China (No.81473279), Science and Technology Innovative Scholar Program of Henan
Province (No.134200510019) and Scientific and Technical Innovation Team of Zhengzhou City (131PCXTD604).

\section{REFERENCES}

1. Wang XQ, Luk JM, Garcia-Barcelo M, Miao X, Leung PP, Ho DW, Cheung ST, Lam BY, Cheung CK, Wong AS, Lau SS, So MT, Yu WC, Cai Q, Liu KS, Hui CK, et al. Liver intestine-cadherin (CDH17) haplotype is associated with increased risk of hepatocellular carcinoma. Clin Cancer Res. 2006; 12:5248-5252.

2. Korobkova EA. Effect of Natural Polyphenols on CYP Metabolism: Implications for Diseases. Chemical research in toxicology. 2015; 28:1359-1390.

3. Ye L, Yang X, Guo E, Chen W, Lu L, Wang Y, Peng X, Yan T, Zhou F and Liu Z. Sorafenib metabolism is significantly altered in the liver tumor tissue of hepatocellular carcinoma patient. PloS one. 2014; 9:e96664.

4. George J, Murray M, Byth K and Farrell GC. Differential alterations of cytochrome $\mathrm{P} 450$ proteins in livers from patients with severe chronic liver disease. Hepatology. 1995; 21:120-128.

5. Iqbal S, Vickers $\mathrm{C}$ and Elias E. Drug metabolism in endstage liver disease. In vitro activities of some phase I and phase II enzymes. Journal of hepatology. 1990; 11:37-42.

6. Pentikainen PJ, Valisalmi L, Himberg JJ and Crevoisier C. Pharmacokinetics of midazolam following intravenous and oral administration in patients with chronic liver disease and in healthy subjects. Journal of clinical pharmacology. 1989; 29:272-277.

7. MacGilchrist AJ, Birnie GG, Cook A, Scobie G, Murray T, Watkinson G and Brodie MJ. Pharmacokinetics and pharmacodynamics of intravenous midazolam in patients with severe alcoholic cirrhosis. Gut. 1986; 27:190-195.

8. Andersson T, Olsson R, Regardh CG and Skanberg I. Pharmacokinetics of [14C] omeprazole in patients with liver cirrhosis. Clinical pharmacokinetics. 1993; 24:71-78.

9. Johnson TN, Boussery K, Rowland-Yeo K, Tucker GT and Rostami-Hodjegan A. A semi-mechanistic model to predict the effects of liver cirrhosis on drug clearance. Clinical pharmacokinetics. 2010; 49:189-206.

10. Zhang H, Gao N, Tian X, Liu T, Fang Y, Zhou J, Wen Q, Xu B, Qi B, Gao J, Li H, Jia L and Qiao H. Content and activity of human liver microsomal protein and prediction of individual hepatic clearance in vivo. Scientific reports. 2015; 5:17671.

11. Edginton AN and Willmann S. Physiology-based simulations of a pathological condition: prediction of pharmacokinetics in patients with liver cirrhosis. Clinical pharmacokinetics. 2008; 47:743-752.

12. Albarmawi A, Czock D, Gauss A, Ehehalt R, Lorenzo Bermejo J, Burhenne J, Ganten TM, Sauer P and Haefeli WE. CYP3A activity in severe liver cirrhosis correlates with Child-Pugh 
and model for end-stage liver disease (MELD) scores. British journal of clinical pharmacology. 2014; 77:160-169.

13. Frye RF, Zgheib NK, Matzke GR, Chaves-Gnecco D, Rabinovitz M, Shaikh OS and Branch RA. Liver disease selectively modulates cytochrome P450--mediated metabolism. Clinical pharmacology and therapeutics. 2006; 80:235-245.

14. Ohnishi A, Murakami S, Akizuki S, Mochizuki J, Echizen $\mathrm{H}$ and Takagi I. In vivo metabolic activity of CYP2C19 and CYP3A in relation to CYP2C19 genetic polymorphism in chronic liver disease. Journal of clinical pharmacology. 2005; 45:1221-1229.

15. Chalasani N, Gorski JC, Patel NH, Hall SD and Galinsky RE. Hepatic and intestinal cytochrome P450 3A activity in cirrhosis: effects of transjugular intrahepatic portosystemic shunts. Hepatology. 2001; 34:1103-1108.

16. Adedoyin A, Arns PA, Richards WO, Wilkinson GR and Branch RA. Selective effect of liver disease on the activities of specific metabolizing enzymes: investigation of cytochromes P450 2C19 and 2D6. Clinical pharmacology and therapeutics. 1998; 64:8-17.

17. Sotaniemi EA, Rautio A, Backstrom M, Arvela P and Pelkonen O. CYP3A4 and CYP2A6 activities marked by the metabolism of lignocaine and coumarin in patients with liver and kidney diseases and epileptic patients. British journal of clinical pharmacology. 1995; 39:71-76.

18. Zhang H, Gao N, Liu T, Fang Y, Qi B, Wen Q, Zhou J, Jia L and Qiao H. Effect of Cytochrome b5 Content on the Activity of Polymorphic CYP1A2, 2B6, and 2E1 in Human Liver Microsomes. PloS one. 2015; 10:e0128547.

19. Bradford MM. A rapid and sensitive method for the quantitation of microgram quantities of protein utilizing the principle of protein-dye binding. Analytical biochemistry. 1976; 72:248-254.

20. Wang XF, Li B, Lan X, Yuan D, Zhang M, Wei YG, Zeng Y, Wen TF, Zhao JC and Yan LN. [Establishment of formula predicting adult standard liver volume for liver transplantation]. Zhonghua wai ke za zhi [Chinese journal of surgery]. 2008; 46:1129-1132.

21. Yuan D, Lu T, Wei YG, Li B, Yan LN, Zeng Y, Wen TF and Zhao JC. Estimation of standard liver volume for liver transplantation in the Chinese population. Transplantation proceedings. 2008; 40:3536-3540.

22. Barter ZE, Tucker GT and Rowland-Yeo K. Differences in cytochrome p450-mediated pharmacokinetics between chinese and caucasian populations predicted by mechanistic physiologically based pharmacokinetic modelling. Clinical pharmacokinetics. 2013; 52:1085-1100.

23. Li GF, Yu G, Liu HX and Zheng QS. Ethnic-specific in vitro-in vivo extrapolation and physiologically based pharmacokinetic approaches to predict cytochrome P450mediated pharmacokinetics in the Chinese population: opportunities and challenges. Clinical pharmacokinetics. 2014; 53:197-202.
24. Vesell ES, Passananti GT, Glenwright PA and Dvorchik $\mathrm{BH}$. Studies on the disposition of antipyrine, aminopyrine, and phenacetin using plasma, saliva, and urine. Clinical pharmacology and therapeutics. 1975; 18:259-272.

25. Raaflaub J and Dubach UC. On the pharmacokinetics of phenacetin in man. European journal of clinical pharmacology. 1975; 8:261-265.

26. Ritschel WA, Brady ME, Tan HS, Hoffmann KA, Yiu IM and Grummich KW. Pharmacokinetics of coumarin and its 7-hydroxy-metabolites upon intravenous and peroral administration of coumarin in man. European journal of clinical pharmacology. 1977; 12:457-461.

27. Findlay JW, Van Wyck Fleet J, Smith PG, Butz RF, Hinton ML, Blum MR and Schroeder DH. Pharmacokinetics of bupropion, a novel antidepressant agent, following oral administration to healthy subjects. European journal of clinical pharmacology. 1981;21:127-135.

28. Kharasch ED, Mitchell D, Coles R and Blanco R. Rapid clinical induction of hepatic cytochrome P4502B6 activity by ritonavir. Antimicrobial agents and chemotherapy. 2008; 52:1663-1669.

29. van Tellingen O, Huizing MT, Panday VR, Schellens JH, Nooijen WJ and Beijnen JH. Cremophor EL causes (pseudo-) non-linear pharmacokinetics of paclitaxel in patients. British journal of cancer. 1999; 81:330-335.

30. Sparreboom A, van Zuylen L, Brouwer E, Loos WJ, de Bruijn P, Gelderblom H, Pillay M, Nooter K, Stoter G and Verweij J. Cremophor EL-mediated alteration of paclitaxel distribution in human blood: clinical pharmacokinetic implications. Cancer research. 1999; 59:1454-1457.

31. Wattanachai N, Polasek TM, Heath TM, Uchaipichat V, Tassaneeyakul W, Tassaneeyakul W and Miners JO. In vitro-in vivo extrapolation of CYP2C8-catalyzed paclitaxel 6alpha-hydroxylation: effects of albumin on in vitro kinetic parameters and assessment of interindividual variability in predicted clearance. European journal of clinical pharmacology. 2011; 67:815-824.

32. Tassaneeyakul W, Veronese ME, Birkett DJ, Doecke CJ, McManus ME, Sansom LN and Miners JO. Co-regulation of phenytoin and tolbutamide metabolism in humans. British journal of clinical pharmacology. 1992; 34:494-498.

33. Wilner KD and Gardner MJ. Tenidap sodium does not alter the clearance or plasma protein binding of tolbutamide in healthy male volunteers. British journal of clinical pharmacology. 1995; 39 Suppl 1:39S-42S.

34. Howgate EM, Rowland Yeo K, Proctor NJ, Tucker GT and Rostami-Hodjegan A. Prediction of in vivo drug clearance from in vitro data. I: impact of inter-individual variability. Xenobiotica; the fate of foreign compounds in biological systems. 2006; 36:473-497.

35. Merz WG and Sandford GR. Isolation and characterization of a polyene-resistant variant of Candida tropicalis. Journal of clinical microbiology. 1979; 9:677-680. 
36. Miners JO, Wing LM, Lillywhite KJ and Smith KJ. Failure of 'therapeutic' doses of beta-adrenoceptor antagonists to alter the disposition of tolbutamide and lignocaine. British journal of clinical pharmacology. 1984; 18:853-860.

37. Dickinson GL, Rezaee S, Proctor NJ, Lennard MS, Tucker GT and Rostami-Hodjegan A. Incorporating in vitro information on drug metabolism into clinical trial simulations to assess the effect of CYP2D6 polymorphism on pharmacokinetics and pharmacodynamics: dextromethorphan as a model application. Journal of clinical pharmacology. 2007; 47:175-186.

38. Naritomi Y, Terashita S, Kimura S, Suzuki A, Kagayama A and Sugiyama Y. Prediction of human hepatic clearance from in vivo animal experiments and in vitro metabolic studies with liver microsomes from animals and humans. Drug metabolism and disposition: the biological fate of chemicals. 2001; 29:1316-1324.

39. LI Zhen HJ-h, JIN Gui-lan. Pharmacokinetic comparison of domestic vs imported omeprazole infusion preparations. Chin Pharm J. 2001; 36:3.

40. Li Zhen HJ-H, Zou Duo-Wu, Li Zhao-Shen, Xu Guo-Ming. Pharmacokinetics and 24-hour Intragastric pH Monitoring after Venous Infusion of Omeprazole in Healthy Subjects. Chin J Clin Pharmacol. 2000; 16:2.

41. Duedahl TH, Dirks J, Petersen KB, Romsing J, Larsen $\mathrm{NE}$ and Dahl JB. Intravenous dextromethorphan to human volunteers: relationship between pharmacokinetics and antihyperalgesic effect. Pain. 2005; 113:360-368.

42. Wang Z, Hall SD, Maya JF, Li L, Asghar A and Gorski JC. Diabetes mellitus increases the in vivo activity of cytochrome P450 2E1 in humans. British journal of clinical pharmacology. 2003; 55:77-85.

43. Moller RA, Fisher JM, Taylor AE, Kolluri S, Gardner MJ, Obach RS and Walsky RL. Effects of steady-state lasofoxifene on CYP2D6- and CYP2E1-mediated metabolism. The Annals of pharmacotherapy. 2006; 40:32-37.

44. Park JY, Kim KA, Park PW and Ha JM. Effect of high-dose aspirin on CYP2E1 activity in healthy subjects measured using chlorzoxazone as a probe. Journal of clinical pharmacology. 2006; 46:109-114.

45. Liangpunsakul S, Kolwankar D, Pinto A, Gorski JC, Hall SD and Chalasani N. Activity of CYP2E1 and CYP3A enzymes in adults with moderate alcohol consumption: a comparison with nonalcoholics. Hepatology. 2005; 41:1144-1150.

46. Prompila N, Wittayalertpanya S and Komolmit P. A study on the pharmacokinetics of chlorzoxazone in healthy Thai volunteers. J Med Assoc Thai. 2007; 90:160-166.

47. Heizmann P, Eckert M and Ziegler WH. Pharmacokinetics and bioavailability of midazolam in man. British journal of clinical pharmacology. 1983; 16 Suppl 1:43S-49S.

48. Yang G, Fu Z, Chen X, Yuan H, Yang H, Huang Y, Ouyang D, Tan Z, Tan H, Huang Z and Zhou H. Effects of the CYP oxidoreductase Ala503Val polymorphism on CYP3A activity in vivo: a randomized, open-label, crossover study in healthy Chinese men. Clinical therapeutics. 2011; 33:2060-2070.

49. Ibrahim A, Karim A, Feldman J and Kharasch E. The influence of parecoxib, a parenteral cyclooxygenase-2 specific inhibitor, on the pharmacokinetics and clinical effects of midazolam. Anesthesia and analgesia. 2002; 95:667-673, table of contents. 